\title{
Generalized connections on affine bundles
}

\author{
T. Mestdag \\ Department of Mathematical Physics and Astronomy, Ghent University \\ Krijgslaan 281 - S9, B-9000 Gent, Belgium \\ email: Tom.Mestdag@UGent.be
}

\begin{abstract}
Connections are among the most important tools to study qualitative features of dynamical systems. In this paper, we discuss generalized connections on affine bundles and show how they appear in the context of Lagrangian systems on affine Lie algebroids.
\end{abstract}

Keywords: affine bundle, Lie algebroid, Lagrangian system, connection

MS:37J99, 53C05, 53C15, 70H03

PACS: 02.40.-k

\section{INTRODUCTION}

Let $\pi: E \rightarrow M$ be an affine bundle, modelled on a vector bundle $\bar{\pi}: \bar{E} \rightarrow M$. Coordinates on $M$ will be denoted by $\left(x^{I}\right)$; adapted coordinates on $E$ by $\left(x^{I}, y^{\alpha}\right)$. Suppose that also an affine map $\rho: E \rightarrow T M$ (with corresponding linear map $\bar{\rho}: \bar{E} \rightarrow T M$ ) is given. We will refer to $\rho$ as the anchor map and use $\rho_{\alpha}^{I}$ and $\rho_{0}^{I}$ for its coordinate functions. This paper concerns the geometric study of dynamical systems on $E$ of the form

$$
\begin{aligned}
\dot{x}^{I} & =\rho_{\alpha}^{I}(x) y^{\alpha}+\rho_{0}^{I}(x), \\
\dot{y}^{\alpha} & =f^{\alpha}(x, y) .
\end{aligned}
$$

Lagrangian systems on so-called 'affine' Lie algebroids motivate our interest in such systems, as we will explain in the next section (see also [6, 7]). Dynamical systems of the form (1) are called 'pseudo-SODEs', where SODE stands for 'second-order ordinary differential equations'. In the special case where the base manifold $M$ is fibred over $\mathbf{R}$, $E=J^{1} M$ is its first jet extension $\left(\left(x^{I}, y^{\alpha}\right)=\left(t, x^{i}, \dot{x}^{i}\right)\right)$ and $\rho$ is the canonical injection $J^{1} M \rightarrow T M$, pseudo-SODEs become indeed a system of second-order differential equations on $M$

$$
\ddot{x}^{i}=f^{i}(t, x, \dot{x}) .
$$

In the following we will often refer to this situation as the 'time-dependent SoDE-case'. Two important tools are extensively used in the geometrical analysis of (time-dependent) SODEs (see $[2,4,5])$ :

1. Every SoDE gives rise to a non-linear connection on $\pi_{M}: J^{1} M \rightarrow M$.

2. This non-linear connection can be linearized to a linear connection, the so-called Berwald-type connection.

This contribution is centred around the following question: Can we generalize the above mentioned tools in such a way that they can be used to study similar questions for the 
dynamical systems (1)? In the time-dependent SoDE-case $\left(E=J^{1} M\right.$ and $\rho$ the canonical injection) the newly found tools should of course correspond to the known situation.

In the next section we discuss pseudo-SODEs of Lagrangian type on affine Lie algebroids. In Section 3 we relate to any pseudo-SODE a section of a prolonged Lie algebroid and we generalize the notion of a connection in such a way that it becomes compatible with the affine anchor map $\rho$. We further show how any pseudo-SODE on an affine Lie algebroid can generate such a generalized connection. In the last section we discuss affine generalized connections and, finally, we define generalized Berwald-type connections. This article is an overview of joint work with W. Sarlet and E. Martínez. Proofs and detailed calculations have been omitted, they can be found in $[9,11,13,14,15]$ and the references therein.

\section{GENERALIZATIONS OF LAGRANGIAN SYSTEMS}

We will first investigate the special subclass of pseudo-SoDEs of the form

$$
\begin{aligned}
\dot{x}^{I} & =\rho_{0}^{I}+\rho_{\alpha}^{I} y^{\alpha} \\
\frac{d}{d t}\left(\frac{\partial L}{\partial y^{\alpha}}\right) & =\rho_{\alpha}^{I} \frac{\partial L}{\partial x^{I}}+\left(C_{0 \alpha}^{\beta}+C_{\gamma \alpha}^{\beta} y^{\gamma}\right) \frac{\partial L}{\partial y^{\beta}},
\end{aligned}
$$

where $L \in C^{\infty}(E)$ and the matrix $\left(\frac{\partial^{2} L}{\partial y^{\alpha} y^{\beta}}\right)$ is supposed to have maximal rank. Obviously, in the time-dependent SODE-case, (3) corresponds to the equations of Lagrangian mechanics. Also here, there is a variational problem at work. Consider curves $\gamma:\left[t_{0}, t_{1}\right] \rightarrow E, t \mapsto(x(t), y(t))$ whose projection on $M$ have fixed endpoints and which satisfy the constraint $\dot{x}^{I}=\rho_{0}^{I}+\rho_{\alpha}^{I} y^{\alpha}$ (i.e. 'admissible' curves). Making use of admissible variations, the equations (3) can be derived from extremizing the functional $J(\gamma)=\int_{t_{0}}^{t_{1}} L(\gamma(t)) d t$, provided the functions $\rho_{\alpha}^{I}, \rho_{0}^{I}, C_{\gamma \alpha}^{\beta}$ and $C_{0 \alpha}^{\beta}$ satisfy the following relations

$$
\rho_{\alpha}^{I} \frac{\partial \rho_{\beta}^{J}}{\partial x^{I}}-\rho_{\beta}^{I} \frac{\partial \rho_{\alpha}^{J}}{\partial x^{I}}=C_{\alpha \beta}^{\gamma} \rho_{\gamma}^{J} \quad \text { and } \quad \rho_{0}^{I} \frac{\partial \rho_{\beta}^{J}}{\partial x^{I}}-\rho_{\beta}^{I} \frac{\partial \rho_{0}^{J}}{\partial x^{I}}=C_{0 \beta}^{\alpha} \rho_{\alpha}^{J} .
$$

These equations are closely related to some of the structure equations of a 'Lie algebroid'. A Lie algebroid is a vector bundle $\tau: \mathrm{V} \rightarrow Q$, which comes equipped with a real Lie bracket on the set of its sections and a linear bundle map $\lambda: \vee \rightarrow T Q$ (and its extension $\lambda: \operatorname{Sec}(\tau) \rightarrow \mathscr{X}(Q))$. Moreover, the bracket and the map $\lambda$ should satisfy, for all $\mathrm{s}, \mathrm{r} \in \operatorname{Sec}(\tau), f \in C^{\infty}(Q)$, the Leibnitz rule $[\mathrm{s}, f \mathrm{r}]=f[\mathrm{~s}, \mathrm{r}]+\lambda(\mathrm{s})(f) \mathrm{r}$.

To understand better the structure (4), we have to extend the notion of a Lie algebroid to affine bundles. Although there are several ways to do so, we will mention here only one. Let $E_{m}$ be a fibre of the affine bundle $\pi$. It is an affine space, modelled on a vector space $\bar{E}_{m}$. We will denote by $E_{m}^{\dagger}=\operatorname{Aff}\left(E_{m}, \mathbb{R}\right)$ the space of affine functions from $E_{m}$ to $\mathbf{R}$. This is a vector space, so we can look at its dual $\tilde{E}_{m}=\left(E_{m}^{\dagger}\right)^{*}$ which is again a vector space, called the bi-dual of $E_{m}$. The collection of all bi-duals gives rise to a vector bundle 
$\tilde{\pi}: \tilde{E} \rightarrow M$, called bi-dual bundle of $\pi$. For example, if $M \rightarrow \mathbf{R}$ and $\pi$ is $\pi_{M}: J^{1} M \rightarrow M$, then $\tilde{\pi}$ is the tangent bundle $\tau_{M}: T M \rightarrow M$.

$\tilde{E}$ contains both a copy of $E$ and $\bar{E}$. Indeed, there exists a canonical injection $\imath: E \rightarrow \tilde{E}$, given by $\boldsymbol{l}_{m}(e)(\phi)=\phi(e)\left(e \in E_{m}, \phi \in \operatorname{Aff}\left(E_{m}, \mathbf{R}\right)\right) . \imath$ is an affine map and we will use $\bar{l}: \bar{E} \rightarrow \tilde{E}$ for its underlying linear map. The above observations lead to a decomposition of elements in $\tilde{E}_{m}$ : For a fixed $e_{0} \in E_{m}$ and for an arbitrary $\tilde{e} \in \tilde{E}_{m}$, there exist $r \in \mathbb{R}$ and $\bar{e} \in \bar{E}_{m}$ such that $\tilde{e}=r l_{m}\left(e_{0}\right)+\bar{\imath}_{m}(\bar{e})$. This property can be used to extend the maps $\rho$ and $\bar{\rho}$ to a linear map $\tilde{\rho}: \tilde{E} \rightarrow T M: \tilde{\rho}_{m}\left(r l\left(a_{0}\right)+\bar{\imath}(\bar{a})\right)=r \rho_{m}\left(a_{0}\right)+\bar{\rho}_{m}(\bar{a})$. If $\left(o,\left\{\bar{e}_{\alpha}\right\}\right)$ is a frame for $\operatorname{Sec}(\pi)$, then $\left\{e_{0}=\imath(o), e_{\alpha}=\bar{l}\left(\bar{e}_{\alpha}\right)\right\}$ is a basis for $\operatorname{Sec}(\tilde{\pi})$. In coordinates, $\tilde{\rho}$ is the map $\left(x^{I}, y^{0}, y^{\alpha}\right) \mapsto\left(\rho_{0}^{I}(x) y^{0}+\rho_{\alpha}^{I}(x) y^{\alpha}\right) \frac{\partial}{\partial x^{l}}$.

There is no need to define structures directly on the affine bundle $\pi$, since we can conveniently make use of the vector bundle structure of $\tilde{\pi}$.

Definition 1 An affine Lie algebroid on $\pi$ is a Lie algebroid on the vector bundle $\tilde{\pi}: \tilde{E} \rightarrow M$ with anchor map $\tilde{\rho}: \tilde{E} \rightarrow T M$ and with a bracket such that, $\forall \zeta, \eta \in \operatorname{Sec}(\pi)$, $[\imath(\zeta), \imath(\eta)] \in \operatorname{Im} \bar{\imath}$.

Locally, the bracket must be of the form

$$
\left[e_{0}, e_{0}\right]=0 \quad\left[e_{0}, e_{\beta}\right]=C_{0 \beta}^{\gamma} e_{\gamma} \quad\left[e_{\alpha}, e_{\beta}\right]=C_{\alpha \beta}^{\gamma} e_{\gamma}
$$

$\left\{e^{0}, e^{\alpha}\right\}$ will denote the basis, dual to $\left\{e_{0}, e_{\alpha}\right\}$. It is easy to see that $e^{0}$ (given by $\left.e^{0}\left(\zeta^{0} e_{0}+\zeta^{\alpha} e_{\alpha}\right)=\zeta^{0}\right)$ is in fact a globally defined 1-form on $\operatorname{Sec}(\tilde{\pi})$. On any Lie algebroid, one can define an exterior derivative (see [8]). It is completely determined by its action on functions and 1 -forms. For an affine Lie algebroid, one finds that

$$
d x^{I}=\rho_{0}^{I} e^{0}+\rho_{\alpha}^{I} e^{\alpha}, \quad d e_{0}=0 \quad \text { and } \quad d e^{\alpha}=-C_{0 \beta}^{\alpha} e^{0} \wedge e^{\beta}-\frac{1}{2} C_{\beta \gamma}^{\alpha} e^{\beta} \wedge e^{\gamma} .
$$

Proposition 1 A Lie algebroid on $\tilde{\pi}$ with anchor $\tilde{\rho}$ is affine if and only if $d e^{0}=0$.

\section{PSEUDO-SODES AND GENERALIZED CONNECTIONS}

We will show next that a Lie algebroid can be 'lifted' to a Lie algebroid structure on a 'prolonged' bundle $\pi^{1}: T^{\tilde{\rho}} E \rightarrow E$ and that it is more convenient to look at (not necessarily Lagrangian) pseudo-SODEs as sections of this bundle, rather than as vector fields on $E$. As announced in the introduction we will generalize the concept of a 'connection' to the current set-up.

The bundle $\pi^{1}$ has been visualized in the diagram. It is a vector bundle whose total manifold is the pullback bundle $\tilde{\rho}^{*} T E$ (i.e. $\left(\tilde{e}, X_{e}\right)$ is an element of $T^{\tilde{\rho}} E$ if $\tilde{\rho}(\tilde{e})=$ $T \pi\left(X_{e}\right)$ ), but whose bundle projection is given by $\pi^{1}\left(\tilde{e}, X_{e}\right)=\tau_{E}\left(X_{e}\right)=e$. Further, $\pi^{2}$ maps the element $\left(\tilde{e}, X_{e}\right) \in T^{\tilde{\rho}} E$ onto $\tilde{e} \in \tilde{E}$, while $\tilde{\rho}^{1}$ projects the same element of $T^{\tilde{\rho}} E$ onto $X_{e}$. Finally, there is also a canonical map $j: T^{\tilde{\rho}} E \rightarrow \pi^{*} \tilde{E},\left(\tilde{e}, X_{e}\right) \mapsto(e, \tilde{e})$. A frame $\left(o,\left\{\bar{e}_{\alpha}\right\}\right)$ for $\operatorname{Sec}(\pi)$ induces a basis $\left\{\mathscr{X}_{0}, \mathscr{X}_{\alpha}, \mathscr{V}_{\alpha}\right\}$ for $\operatorname{Sec}\left(\pi^{1}\right)$, where (with $A=0, \alpha$ )

$$
\mathscr{X}_{A}(e)=\left(e_{A}(\pi(e)),\left.\rho_{A}^{I}(x) \frac{\partial}{\partial x^{I}}\right|_{e}\right) \quad \text { and } \quad \mathscr{V}_{\alpha}(e)=\left(\mathrm{o}(\pi(e)),\left.\frac{\partial}{\partial y^{\alpha}}\right|_{e}\right) \text {. }
$$




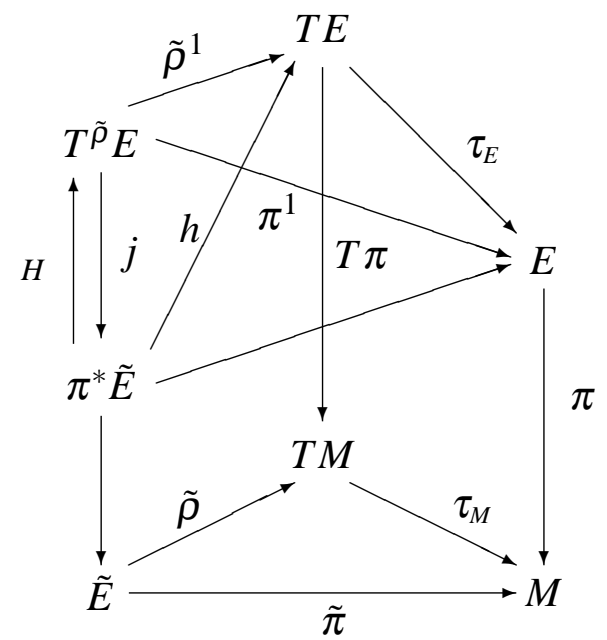

FIGURE 1. The prolonged bundle $\pi^{1}$.

Remark that $\tilde{\rho}^{1}\left(\zeta^{0} \mathscr{X}_{0}+\zeta^{\alpha} \mathscr{X}_{\alpha}+Z^{\alpha} \mathscr{V}_{\alpha}\right)=\left(\rho_{0}^{I} \zeta^{0}+\rho_{\alpha}^{I} \zeta^{\alpha}\right) \frac{\partial}{\partial x^{I}}+Z^{\alpha} \frac{\partial}{\partial y^{\alpha}}$.

It is well-known that a connection on a vector bundle $\tau: \mathrm{V} \rightarrow M$ selects a (horizontal) distribution which is complementary to the set of vertical elements in $T \bigvee$. For the bundle $\pi^{1}$ too, we can identify a set of 'vertical' vectors: they are those elements of $T^{\tilde{\rho}} E$ whose projection on $\tilde{E}$ (by means of $\pi^{2}$ ) vanishes. The set of all such verticals is denoted by $\operatorname{Ver}\left(\pi^{1}\right)$. A section $\mathscr{Z} \in \operatorname{Sec}\left(\pi^{1}\right)$ is vertical if its image lies in $\operatorname{Ver}\left(\pi^{1}\right)$, or, locally, if it lies in the span of the sections $\mathscr{V}_{\alpha}$. The required extension of the concept 'connection' will generate a direct complement of $\operatorname{Ver}\left(\pi^{1}\right)$ within $T^{\tilde{\rho}} E$. As usual, the definition can be cast in terms of a horizontal lift.

Definition $2 A$ (generalized) $\tilde{\rho}$-connection on $\pi$ is a map ${ }^{H}: \pi^{*} \tilde{E} \rightarrow T^{\tilde{\rho}} E$ for which $j \circ^{H}=i d_{\pi^{*} \tilde{E}}$ holds. Then $T^{\tilde{\rho}} E=\operatorname{Im}{ }^{H} \oplus \operatorname{Ver}\left(\pi^{1}\right)$.

In what follows we will usually refer to a $\tilde{\rho}$-connection in terms of its associated map $h:=\tilde{\rho}^{1} \circ^{H}: \pi^{*} \tilde{E} \rightarrow T E$. Locally, a generalized connection is completely determined by a set of connection coefficients $\Gamma_{0}^{\beta}, \Gamma_{\alpha}^{\beta} \in C^{\infty}(E)$. If $\tilde{X}=X^{0} e_{0}+X^{\alpha} e_{\alpha} \in \operatorname{Sec}\left(\pi^{*} \tilde{\pi}\right)$, then $\tilde{X}^{H}=X^{0} \mathscr{H}_{0}+X^{\alpha} \mathscr{H}_{\alpha} \in \operatorname{Sec}\left(\pi^{1}\right)$ with $\mathscr{H}_{0}=e_{0}{ }^{H}=\mathscr{X}_{0}-\Gamma_{0}^{\beta} \mathscr{V}_{\beta}$ and $\mathscr{H}_{\alpha}=e_{\alpha}{ }^{H}=$ $\mathscr{X}_{\alpha}-\Gamma_{\alpha}^{\beta} \mathscr{V}_{\beta}$.

Let us now come back to the dynamical systems (1). To any pseudo-SODE we can associate a section $\Gamma$ of $\pi^{1}$ with the property that $\pi^{2} \circ \Gamma=\imath$. Indeed, such sections are locally of the form $\Gamma=\mathscr{X}_{0}+y^{\alpha} \mathscr{X}_{\alpha}+f^{\alpha} \mathscr{V}_{\alpha}$ and can thus represent pseudo-SoDEs in this framework. Remark that $\tilde{\rho}^{1}(\Gamma)$ is then exactly the vector field whose integral curves are solutions of (1).

Proposition 2 Every pseudo-SoDE $\Gamma=\mathscr{X}_{0}+y^{\alpha} \mathscr{X}_{\alpha}+f^{\alpha} \mathscr{V}_{\alpha}$ on a affine Lie algebroid gives rise to a $\tilde{\rho}$-connection with connection coefficients

$$
\Gamma_{\beta}^{\alpha}=-\frac{1}{2}\left(\frac{\partial f^{\alpha}}{\partial y^{\beta}}+y^{\gamma} C_{\gamma \beta}^{\alpha}+C_{0 \beta}^{\alpha}\right) \quad \text { and } \quad \Gamma_{0}^{\alpha}=-f^{\alpha}-y^{\beta} \Gamma_{\beta}^{\alpha} .
$$


The above theorem generalizes the well-known non-linear connection of a SODE which has been mentioned in the introduction (see e.g. [3]). We can also give a more intrinsic formulation of this proposition. Let $\left\{\mathscr{X}^{0}, \mathscr{X}^{\alpha}, \mathscr{V}^{\alpha}\right\}$ be the basis of $\operatorname{Sec}\left(\left(\pi^{1}\right)^{*}\right)$ which is dual to $\left\{\mathscr{X}_{0}, \mathscr{X}_{\alpha}, \mathscr{V}_{\alpha}\right\}$. Then, $\mathscr{X}^{0}$ is a global 1 -form on $\operatorname{Sec}\left(\pi^{1}\right)$ and $S=\left(\mathscr{X}^{\alpha}-\right.$ $\left.y^{\alpha} \mathscr{X}^{0}\right) \otimes \mathscr{V}_{\alpha}$, the vertical endomorphism, a globally defined vector-valued 1 -form on $\operatorname{Sec}\left(\pi^{1}\right)$. Moreover, it is possible to prolong the Lie algebroid structure of $\pi$ to the bundle $\pi^{1}$.

Proposition 3 An (affine) Lie algebroid on $\tilde{\pi}$ with anchor $\tilde{\rho}$ can be extended to an (affine) Lie algebroid on $\pi^{1}$ with anchor $\tilde{\rho}^{1}$ and Lie bracket $\left[\mathscr{X}_{\alpha}, \mathscr{X}_{\beta}\right]=C_{\alpha \beta}^{\gamma} \mathscr{X}_{\gamma}$, $\left[\mathscr{X}_{0}, \mathscr{X}_{\beta}\right]=C_{0 \beta}^{\gamma} \mathscr{X}_{\gamma},\left[\mathscr{V}_{\alpha}, \mathscr{X}_{\beta}\right]=0,\left[\mathscr{X}_{0}, \mathscr{V}_{\beta}\right]=0$ and $\left[\mathscr{V}_{\alpha}, \mathscr{V}_{\beta}\right]=0$.

If $d$ is the exterior derivative of this extended Lie algebroid (which acts on forms of $\operatorname{Sec}\left(\pi^{1}\right)$ ), then $d_{\Gamma}=\left[i_{\Gamma}, d\right]$ is a degree zero derivation, which extends to vector-valued forms in the usual way. The horizontal part of a $\mathscr{Z} \in \operatorname{Sec}\left(\pi^{1}\right)$ for the connection in Prop. 2 is then given by $\frac{1}{2}\left(I-d_{\Gamma} S+\mathscr{X}^{0} \otimes \Gamma\right)(\mathscr{Z})$.

Remark that $\Gamma \in \operatorname{Sec}\left(\pi^{1}\right)$ is a pseudo-SoDE if and only if $S(\Gamma)=0$ and $\left\langle\Gamma, \mathscr{X}^{0}\right\rangle=1$. In particular, if $d$ is again the exterior derivative of the lifted Lie algebroid, we can define for a function $L \in C^{\infty}(E)$ a 1 -form $\theta_{L}=S(d L)+L \mathscr{X}^{0}$ on $\operatorname{Sec}\left(\pi^{1}\right)$. The pseudo-SoDE, associated to the Lagrangian system (3), is then a solution of the equation $i_{\Gamma} d \theta_{L}=0$.

\section{AFFINE $\tilde{\rho}$-CONNECTIONS AND BERWALD-TYPE CONNECTIONS}

A special subclass of $\tilde{\rho}$-connections on an affine bundle is formed by those for which the connection coefficients are affine functions, i.e. $\Gamma_{0}^{\alpha}(x, y)=\Gamma_{00}^{\alpha}(x)+\Gamma_{0 \beta}^{\alpha}(x) y^{\beta}$ and $\Gamma_{\mu}^{\alpha}(x, y)=\Gamma_{\mu 0}^{\alpha}(x)+\Gamma_{\mu \beta}^{\alpha}(x) y^{\beta}$. Before we can characterize such connections, we need to say a few words about 'linear' generalized connections (see also [1]).

It is not difficult to introduce also 'generalized' connections for the vector bundle $\bar{\pi}$ on which $\pi$ is modelled. Indeed, by replacing the affine bundle $\pi$ with the vector bundle $\bar{\pi}$ in the diagram, we arrive at a new prolonged vector bundle $\bar{\pi}^{1}: T^{\tilde{\rho}} \bar{E} \rightarrow E$. Using the corresponding $\bar{j}: T^{\tilde{\rho}} \bar{E} \rightarrow \bar{\pi}^{*} \tilde{E}$, it is easy to give a well-defined meaning to the concept of a $\tilde{\rho}$-connection $\bar{h}: \bar{\pi}^{*} \tilde{E} \rightarrow T \bar{E}$ on $\bar{\pi}$. When in addition the connection coefficients are linear functions, we will call the connection 'linear'. Putting $\bar{\Sigma}\left(\bar{e}_{1}, \bar{e}_{2}\right)=\bar{e}_{1}+\bar{e}_{2}$, for $\bar{e}_{1}, \bar{e}_{2} \in \bar{E}_{m}$, a more intrinsic formulation of this property is the following: a $\tilde{\rho}$-connection $\bar{h}$ on $\bar{\pi}$ is linear if and only if

$$
\bar{h}\left(\bar{e}_{1}+\bar{e}_{2}, \tilde{e}\right)=T_{\left(\bar{e}_{1}, \bar{e}_{2}\right)} \bar{\Sigma}\left(\bar{h}\left(\bar{e}_{1}, \tilde{e}\right), \bar{h}\left(\bar{e}_{2}, \tilde{e}\right)\right),
$$

It is easy to find an equivalent characterization in terms of a covariant derivative operator. One can check that the existence of a linear $\bar{h}$ is equivalent with the existence of an operator $\bar{\nabla}: \operatorname{Sec}(\tilde{\pi}) \times \operatorname{Sec}(\bar{\pi}) \rightarrow \operatorname{Sec}(\bar{\pi}):(\tilde{\zeta}, \bar{\eta}) \mapsto \bar{\nabla}_{\tilde{\zeta}} \bar{\eta}$ which is R-linear in both its arguments and satisfies

$$
\bar{\nabla}_{f \tilde{\zeta}} \bar{\eta}=f \bar{\nabla}_{\tilde{\zeta}} \bar{\eta} \quad \text { and } \quad \bar{\nabla}_{\tilde{\zeta}}(f \bar{\eta})=f \bar{\nabla}_{\tilde{\zeta}} \bar{\eta}+\tilde{\rho}(\tilde{\zeta})(f) \bar{\eta}, \quad\left(f \in C^{\infty}(M)\right)
$$


Generalized connections on $\pi$ with affine coefficients have properties which are similar to those of linear connections. Let $\Sigma(e, \bar{e})=e+\bar{e}$, for $e \in E_{m}$ and $\bar{e} \in \bar{E}_{m}$.

Proposition 4 A $\tilde{\rho}$-connection $h$ on $\pi$ is affine, if there exists a linear $\tilde{\rho}$-connection $\bar{h}$ on $\bar{\pi}$, which is related to $h$ in such a way that

$$
h(e+\bar{e}, \tilde{e})=T_{(e, \bar{e})} \Sigma(h(e, \tilde{e}), \bar{h}(\bar{e}, \tilde{e})) .
$$

Equivalently, a $\tilde{\rho}$-connection on $\pi$ is affine if there exists an operator $\nabla: \operatorname{Sec}(\tilde{\pi}) \times$ $\operatorname{Sec}(\pi) \rightarrow \operatorname{Sec}(\bar{\pi}):(\tilde{\zeta}, \eta) \mapsto \nabla_{\tilde{\zeta}} \eta$ which is $\mathbf{R}$-linear in its first argument, satisfies $\nabla_{f \tilde{\zeta}} \sigma=f \nabla_{\tilde{\zeta}} \sigma$ and is related to a covariant derivative operator $\bar{\nabla}$ of a linear $\tilde{\rho}-$ connection on $\bar{\pi}$ by means of

$$
\nabla_{\tilde{\zeta}}(\sigma+f \bar{\eta})=\nabla_{\tilde{\zeta}} \sigma+f \bar{\nabla}_{\tilde{\zeta}} \bar{\eta}+\tilde{\rho}(\tilde{\zeta})(f) \bar{\eta}
$$

It is possible to give an explicit relation between the horizontal lift $h$ and the operator $\nabla$. First, we need to define the vertical lift: it is the map $v: \operatorname{Sec}\left(\pi^{*} \tilde{\pi}\right) \rightarrow \mathscr{X}(E)$ given by $v\left(X^{0} e_{0}+X^{\alpha} e_{\alpha}\right)=\left(X^{\alpha}-y^{\alpha} X^{0}\right) \frac{\partial}{\partial y^{\alpha}}$. There is also an inverse map for vertical vector fields: $\left(Y^{\alpha} \frac{\partial}{\partial y^{\alpha}}\right)_{v}=Y^{\alpha} e_{\alpha} \in \operatorname{Sec}\left(\pi^{*} \tilde{\pi}\right)$. It is not difficult to see that the brackets $[h(\tilde{\zeta}), v(\bar{\sigma})]$ and $[h(\tilde{\zeta}), v(\sigma)]$ of vector fields on $E$ are vertical. $\tilde{\zeta} \in \operatorname{Sec}(\tilde{\pi}), \bar{\sigma} \in \operatorname{Sec}(\bar{\pi})$ and $\sigma \in \operatorname{Sec}(\pi)$ have here been identified with 'basic' sections of $\operatorname{Sec}\left(\pi^{*} \tilde{\pi}\right)$. A section $\tilde{X}$ of $\pi^{*} \tilde{\pi}$ is basic if it is of the form $e \mapsto(e, \tilde{\zeta})$ for a certain $\tilde{\zeta} \in \operatorname{Sec}(\tilde{\pi})$. If the connection is affine, then also $[h \tilde{\zeta}, v(\bar{\sigma})]_{v}$ and $[h \tilde{\zeta}, v(\sigma)]_{v}$ are basic sections and one can prove that

$$
\bar{\nabla}_{\tilde{\zeta}} \bar{\sigma}=[h \tilde{\zeta}, v(\bar{\sigma})]_{v} \quad \text { and } \quad \nabla_{\tilde{\zeta}} \sigma=[h \tilde{\zeta}, v(\sigma)]_{v}
$$

The connection coefficients of the connection $\bar{h}$ in Prop. 4 can be found in the linear part of those of $h$, i.e. $\bar{\Gamma}_{0}^{\alpha}(x, w)=\Gamma_{0 \beta}^{\alpha}(x) w^{\beta}$ and $\bar{\Gamma}_{\mu}^{\alpha}(x, w)=\Gamma_{\mu \beta}^{\alpha}(x) w^{\beta}$. An other associated connection is the one whose coefficients are $\tilde{\Gamma}_{0}^{\alpha}\left(x, y^{0}, y\right)=\Gamma_{00}^{\alpha}(x) y^{0}+\Gamma_{0 \beta}^{\alpha}(x) y^{\beta}$ and $\tilde{\Gamma}_{\mu}^{\alpha}\left(x, y^{0}, y\right)=\Gamma_{\mu 0}^{\alpha}(x) y^{0}+\Gamma_{\mu \beta}^{\alpha}(x) y^{\beta}$. This linear $\tilde{\rho}$-connection on $\tilde{\pi}$ is related to $h$ as follows:

Proposition 5 is affine, if there exists a linear $\tilde{\rho}$-connection $\tilde{h}: \tilde{\pi}^{*} \tilde{E} \rightarrow T \tilde{E}$ on $\tilde{\pi}: \tilde{E} \rightarrow$ $M$ such that,

$$
\tilde{h} \circ \imath=T \imath \circ h .
$$

There is, of course, also a corresponding derivative operator $\tilde{\nabla}: \operatorname{Sec}(\tilde{\pi}) \times \operatorname{Sec}(\tilde{\pi}) \rightarrow$ $\operatorname{Sec}(\tilde{\pi})$.

Proposition 6 A linear $\tilde{\rho}$-connection on $\tilde{\pi}$ is associated with an affine $\tilde{\rho}$-connection on $\pi$ if and only if $e^{0}$ is parallel w.r.t. $\tilde{\nabla}$.

We are now ready to define Berwald-type connections. In the time-dependent SoDEcase, they are linearized versions of an originally non-linear connection on $\pi_{M}$. The price to pay is that the bundle on which they live is a little bit more complicated: 
usually Berwald-type connections are defined on the (pull-back) vector bundle $\pi_{M}^{*} \tau_{M}$ as a covariant derivative operator. However, it can already be noticed in the time-dependent case that this operator is in fact of the type $\tilde{\nabla}$ and thus associated to an affine connection $\nabla$ on the affine bundle $\pi_{M}^{*} \pi_{M}$. In the general set-up, Berwald-type connections will therefore be affine $\tilde{\rho}^{1}$-connections on the affine bundle $\pi^{*} \pi: \pi^{*} E \rightarrow E$. We will explain below that the affine bundle does not need to have the structure of an affine Lie algebroid for this purpose.

We briefly explain two ways to define them. First, it is easy to see that an affine connection $\mathrm{D}: \operatorname{Sec}\left(\pi^{1}\right) \times \operatorname{Sec}\left(\pi^{*} \pi\right) \rightarrow \operatorname{Sec}\left(\pi^{*} \pi\right)$ is completely determined by its action on horizontal and vertical lifts of basic sections in its first argument and its action on basic sections in its second argument. In the particular case that the $\tilde{\rho}$-connection one starts from is already affine, the Berwald-type connections should essentially reproduce a copy of themselves. Therefore, we can find inspiration in the relations (5) and put

$$
\mathrm{D}_{\tilde{\zeta} H} \sigma=[h \tilde{\zeta}, v \sigma]_{v}, \quad \overline{\mathrm{D}}_{\tilde{\zeta}^{H}} \bar{\sigma}=[h \tilde{\zeta}, v \bar{\sigma}]_{v}, \quad \mathrm{D}_{\bar{\eta}^{V}} \sigma=\overline{\mathrm{D}}_{\bar{\eta}^{V}} \bar{\sigma}=0 .
$$

One can easily check that this definition is consistent with the module structure over $C^{\infty}(M)$ and that the above operator can be extended to arbitrary sections in the obvious way.

The second method uses the fact that the connection is completely determined by specifying the rule of parallel transport along two specific classes of admissible curves in $T^{\tilde{\rho}} E$. A detailed analysis in [13] revealed that, for the subclass of vertical curves, there are in fact two 'natural' ways to fix such a rule, leading thus to two different Berwaldtype connections. We will not go deeper into this matter here; we will only give the defining relations for this second Berwald-type connection D.

$$
\hat{\mathrm{D}}_{\tilde{\zeta}^{H}} \sigma=[h \tilde{\zeta}, v \sigma]_{v}, \quad \overline{\hat{\mathrm{D}}}_{\tilde{\zeta}^{H}} \bar{\sigma}=[h \tilde{\zeta}, v \bar{\sigma}]_{v}, \quad \hat{\mathrm{D}}_{\bar{\eta}^{V}} \sigma=-\bar{\eta}, \quad \overline{\hat{\mathrm{D}}}_{\bar{\eta}^{v}} \bar{\sigma}=0 .
$$

A similar behaviour was detected before in the time-dependent SoDE-case: the first connection corresponds with the one in [4], while the second one is given in [12] and is basically the same one as in [10]. We now list the coordinate expression (for the associated operator $\tilde{D}$ ). Both connections share the expressions (with $A=0, \alpha$ ),

$$
\tilde{\mathrm{D}}_{\mathscr{H}_{A}} e_{0}=\left(\Gamma_{A}^{\gamma}-y^{\beta} \frac{\partial \Gamma_{A}^{\gamma}}{\partial y^{\beta}}\right) e_{\gamma}, \quad \tilde{\mathrm{D}}_{\mathscr{H}_{A}} e_{\beta}=\frac{\partial \Gamma_{A}^{\gamma}}{\partial y^{\beta}} e_{\gamma}, \quad \tilde{\mathrm{D}}_{\mathscr{V}_{\alpha}} e_{\beta}=0
$$

while for the first $\tilde{\mathrm{D}}_{\mathscr{V}_{\alpha}} e_{0}=0$ and for the second $\tilde{\hat{\mathrm{D}}}_{\mathscr{V}_{\alpha}} e_{0}=-e_{\alpha}$. Unlike in the timedependent SODE-case, there are no direct defining formulas for the covariant derivatives. Such formulas become available, however, when the affine bundle $\pi: E \rightarrow M$ has the structure of an affine Lie algebroid. Remark that it is clear from the coordinate expressions that such an additional structure is not required to define Berwald-type connections.

Some final comments: Berwald-type connections have been successfully applied in a number of applications concerning qualitative feature of SODEs. A few examples of 
such problems are mentioned below (see also $[2,4,5]$ ):

- Does a coordinate transformation $x^{i}=x^{i}(t, \bar{x})$ exist, such that the system (2) becomes linear (i.e. of the form $\ddot{\bar{x}}^{j}=A_{k}^{j}(t) \bar{x}^{k}+B_{k}^{j}(t) \dot{\bar{x}}^{k}+C^{j}(t)$ )?

- Can we construct a Lagrangian for this system?

- What are the conditions for the existence of a coordinate transformation in which (2) completely decouples?

It is our belief that the above 'generalized' Berwald-type connections will prove to be equally useful to answer similar questions for pseudo-SODEs.

\section{REFERENCES}

1. F. Cantrijn and B. Langerock, Generalised connections over a bundle map, Diff. Geom. and its Appl. 18 (2003), 295-317.

2. F. Cantrijn, W. Sarlet, A. Vandecasteele and E. Martínez, Complete separability of time-dependent second-order ordinary differential equations, Acta Appl. Math. 42 (1996), 309-334.

3. M. Crampin, Jet bundle techniques in analytical mechanics, Quaderni del consiglio nazionale delle ricerche, gruppo nazionale di fisica matematica 47 (1995).

4. M. Crampin, E. Martínez and W. Sarlet, Linear connections for systems of second-order ordinary differential equations, Ann. Inst. H. Poincaré, Phys. Théor. 65 (1996), 223-249.

5. M. Crampin, W. Sarlet, E. Martínez, G.B. Byrnes and G.E. Prince, Towards a geometrical understanding of Douglas's solution of the inverse problem of the calculus of variations, Inverse problems $\mathbf{1 0}$ (1994), 245-260.

6. K. Grabowska, J. Grabowski and P. Urbañski, Lie brackets on affine bundles, Ann. Glob. Anal. Geom. 24 (2003), 101-130.

7. K. Grabowska, J. Grabowski and P. Urbañski, AV-differential geometry: Poisson and Jacobi structures, preprint 2004 (math.DG/0402435).

8. K. Mackenzie, Lie groupoids and Lie algebroids in differential geometry, London Math. Soc. Lect. Notes Series 124 (Cambridge Univ. Press, 1987).

9. E. Martínez, T. Mestdag and W. Sarlet, Lie algebroid structures and Lagrangian systems on affine bundles, J. Geom. Phys. 44 (2002), 70-95.

10. E. Massa and E. Pagani, Jet bundle geometry, dynamical connections, and the inverse problem of Lagrangian mechanics, Ann. Inst. H. Poincaré Phys. Théor. 61 (1994), 17-62.

11. T. Mestdag, Generalized connections on affine Lie algebroids, Rep. on Math. Phys. 51 (2003), 297305.

12. T. Mestdag and W. Sarlet, The Berwald-type connection associated to time-dependent second-order differential equations, Houston J. Math., 27 (2001), 763-797.

13. T. Mestdag and W. Sarlet, The Berwald-type linearization of generalized connections, J. Phys. A 36 (2003), 8049-8069.

14. T. Mestdag, W. Sarlet and E. Martínez, Note on generalized connections and affine bundles, J. Phys. A 35 (2002), 9843-9856.

15. W. Sarlet, T. Mestdag and E. Martínez, Lie algebroid structures on a class of affine bundles, J. Math. Phys. 43 (2002), 5654-5674. 